\title{
AVALIAÇÃO DOS TRANSPLANTES HEPÁTICOS NO HOSPITAL SANTA ISABEL DE BLUMENAU/SC, NO PERÍODO DE JANEIRO DE 2009 A MAIO DE 2015
}

\author{
Assessment of the liver transplantation in Hospital Santa Isabel of Blumenau/SC \\ from January, 2009 to May, 2015
}

\author{
Marcelo Augusto Scheidemantel Nogara', Camila Ribeiro Batista², Juliana Tasso Candido de Lima²
}

\section{RESUMO}

Objetivo: Avaliar os transplantados hepáticos no Hospital Santa Isabel, centro único de transplantes em Blumenau/ SC, no período de janeiro de 2009 a maio de 2015. Métodos: Trata-se de um estudo de coorte retrospectivo, com pacientes submetidos a transplante hepático no Hospital Santa Isabel de Blumenau. O estudo foi realizado através de coleta de dados secundários, através dos prontuários dos pacientes, durante sua permanência hospitalar para o transplante. Foram avaliados os dados epidemiológicos (idade, sexo, cidade de origem e indicação do transplante hepático), escore MELD, causas de óbito e evolução desses transplantados (ocorrência de retransplante, sobrevida e tempo de internação em UTI). Resultados: O total foi de 614 pacientes, com média de idade de 52,23 anos. Sexo masculino foi prevalente (74,91\%). Quanto à procedência, $11,4 \%$ eram de Blumenau; $7,98 \%$, de Florianópolis/ SC e $22,25 \%$, de outros estados. Do total de pacientes, $55,86 \%$ possuíam MELD maior ou igual a 18 . A principal indicação para transplante foi cirrose por hepatite C (25,6\%) e carcinoma hepatocelular (18,6\%). A maior parte dos pacientes $(70,4 \%)$ permaneceu entre 0 e 5 dias na UTI. As principais causas de óbito nos seis anos observados foram de $35,93 \%$, sem considerar os retransplantes, e as principais causas de óbito nos seis anos observados, foram: causas gerais (34,93\%), a partir das desordens cardiovasculares terem sido prevalentes $(91,25 \%)$, seguidas de causas cerebrovasculares (3,75\%), insuficiência múltipla de órgãos $(3,75 \%)$ e causas renais $(1,25 \%)$; choque séptico $(16,59 \%)$, desordens da coagulação (5,24\%), não funcionamento primário (3,93\%) e causas não informadas (35,8\%). A taxa de sobrevida em um e cinco anos, foi, respectivamente, de 67,94\% e 60,70\%. Conclusão: Evidenciaramse dados semelhantes aos da literatura, comprovando ser este um serviço em evolução, com casuística de bons resultados.

Descritores: Transplante de Fígado; Taxa de Sobrevida; Prognóstico; Insuficiência Hepática; Cirrose Hepática.

Instituições:

${ }^{1}$ Serviço de Gastroenterologia, Hepatologia e Cirurgia Geral Equipe de Transplantes do Hospital Santa Isabel, Blumenau/SC, Brasil.

${ }^{2}$ Curso de Medicina da Universidade Regional de Blumenau FURB; Blumenau/SC, Brasil.

\section{Correspondência:}

Marcelo Augusto Scheidemantel Nogara.

Rua Floriano Peixoto, 300, s/201 - CEP 89010-906 - Blumenau/SC Tel/fax: (47) 3322-1205.

E-mail: kmnogara@terra.com.br

Recebido em: 15/06/2015

Aceito em: 30/06/2015

\section{INTRODUÇÃO}

O transplante hepático é considerado a melhor terapia para pacientes hepatopatas crônicos em condição terminal. ${ }^{1}$ As principais indicações para transplante são: cirrose por hepatite $C$, doença colestática, cirrose alcoólica, tumor hepático primário e doença hepática aguda. ${ }^{2}$ Além do benefício da sobrevivência, o transplante de fígado promove uma melhoria na qualidade de vida. ${ }^{3}$ O primeiro transplante hepático foi realizado em 1963; desde então, o número de cirurgias vem aumentando significativamente. ${ }^{4}$ Segundo a Associação Brasileira de Transplantes de Órgãos, em 2004 foram realizados 959 transplantes no Brasil, e, após dez anos, houve aumento 
de $83 \%$ dos transplantes hepáticos. Em 2014, do total de transplantes de fígado no Brasil, 113 foram realizados no estado de Santa Catarina. ${ }^{5}$

Com os cuidados perioperatórios, as novas técnicas cirúrgicas, a melhor seleção de receptores, a imunossupressão clínica e a melhor gestão de doadores, aproximadamente $90 \%$ dos pacientes sobrevivem ao primeiro ano pós-operatório. Porém, estudos demonstram, universalmente, que a sobrevivência em longo prazo não melhorou. ${ }^{1,6}$ Entre as causas de mortalidade tardia, as mais comuns foram: malignidade, eventos cardíacos, falência do sistema múltiplo, infecção e falência do enxerto. ${ }^{7}$

Considerando tais aspectos, é possível presumir que nesta década haverá aumento progressivo do número de transplantes hepáticos. Sendo esse procedimento um dos mais complexos da cirurgia moderna, é importante analisar os fatores que determinam o bom andamento do transplante e seu prognóstico.

\section{OBJETIVO}

O objetivo do presente trabalho foi avaliar os transplantados hepáticos em um centro único de transplantes em Blumenau/SC - Hospital Santa Isabel, no período de janeiro de 2009 a maio de 2015.

\section{MATERIAIS E MÉTODOS}

Trata-se de uma coorte retrospectiva, em que o critério de inclusão seria o paciente ter sido submetido ao transplante hepático no estado de Santa Catarina e que este tivesse sido realizado no Hospital Santa Isabel de Blumenau - SC. Pacientes que realizaram a cirurgia após maio de 2015 foram excluídos da pesquisa.

O estudo será feito através de coleta de dados secundários, através dos prontuários dos pacientes no período de internação hospitalar para o transplante. Os dados epidemiológicos (idade em anos, sexo, cidade de origem, indicação do transplante hepático); escore MELD; tempo de espera na fila de transplantes, causas de óbito e evolução desses transplantados (sobrevida e tempo de internação em UTI) serão pesquisados.

Para análise estatística, a idade será considerada uma variável contínua, da qual será avaliada sua média. As variáveis sexo, cidade de origem, indicação do transplante, escore MELD pré-transplante, avaliado pelo valor $<18$ ou $\geq 18$, pois estudos comprovam que, com pontuação acima de 18, o paciente possui hepatopatia grave e o transplante trará ganho na sobrevida. ${ }^{8}$ Tempo de espera na fila de transplantes em dias e tempo de internação em UTI em dias de permanência serão avaliados para possíveis associações com a variável de desfecho: sobrevida.
Para associarem as variáveis, foi utilizado o Teste Qui quadrado de independência e para a comparação dos grupos, utilizou-se o Teste $t$ de Student e o Teste $F$ de análise de variância (ANOVA). As probabilidades de sobrevida foram calculadas de acordo com o método proposto por Kaplan-Meier. O teste de Log-Rank foi empregado para a comparação das funções de sobrevida (atributos) para cada variável. Em todos os testes utilizados, considerou-se a significância com o valor $P<0,05$. $A$ análise dos dados foi realizada pelo software Microsoft Excel 2013 e software Statistica versão 7 de 2004 .

Os pacientes não serão identificados de qualquer forma, respeitando o termo de compromisso de utilização e divulgação de dados de acordo com as normas vigentes expressas na Resolução n¹96/1996 do Conselho Nacional de Saúde/Ministério da Saúde. As informações e dados terão sua confidencialidade e privacidade asseguradas.

\section{RESULTADOS}

Totalizaram-se 614 pacientes transplantados, sendo que os retransplantes foram inclusos na amostra $(n=24)$. Durante os seis anos observados, constatou-se que o sexo masculino foi majoritário (74,91\%). Em 2011, houve maior quantidade de pacientes, representando $16,77 \%$ do total de transplantes. A média de idade dos pacientes foi estabelecida em 52,23 anos, tendo sido maior para os homens (53,34 vs 48,92 anos). Observou-se que as idades apresentaram maior dispersão, descritivamente no sexo feminino, com $30,82 \%$. Para os homens, a idade foi considerada mais regular. Dos pacientes, $70,28 \%$ obtiveram MELD com pontuação acima de 18 .

Em relação à origem dos pacientes, $77,5 \%$ eram oriundos do estado de Santa Catarina e $22,5 \%$ eram de outros estados do Brasil. A partir dos pacientes originários de Santa Catarina, $14,70 \%$ eram de Blumenau, 10,29\% eram de Florianópolis e 8,4\%, de Criciúma.

Dentre as causas para a realização do transplante hepático, a hepatite C foi mais frequente, com $24,6 \%$ dos casos, seguido de carcinoma hepatocelular (17,9\%) e cirrose alcoólica (16,3\%). A indicação de transplante por carcinoma hepatocelular abrangeu pacientes infectados pelos vírus B e C. Daqueles, $57,27 \%$ $(n=63)$ possuiam diagnóstico de infecção pelo vírus $C$, enquanto que $10 \% \quad(n=11)$ possuiam diagnóstico de infecção pelo vírus $B$. Ao considerar os pacientes com diagnóstico de hepatocarcinoma infectados pelo vírus $\mathrm{C}$ e pacientes com infecção desse vírus de forma isolada, totalizam-se 214 , ou seja, $34,85 \%$ do total dos transplantes hepáticos. 
Em relação ao total de dias de permanência em UTI, foi preponderante o período de zero a cinco dias $(70,4 \%)$, sendo que $66,8 \%$ destes foram a óbito nesse período. No mais, $18,2 \%$ permaneceram de seis a 10 dias na UTI e o restante permaneceu mais de 10 dias $(11,4 \%)$. $O$ tempo de sobrevida dos pacientes sofre decréscimo de acordo com o maior período em UTI. Os pacientes que ficaram de zero a cinco dias, tiveram sobrevida de 2,29 anos, seis a 10 dias, 2,14 anos; e 11 a 15 dias, 2,07 anos. $O$ índice de letalidade, dentre os que realizaram transplante durante o período observado, foi de $35,93 \% \quad(n=212)$, sem considerar os retransplantes, que possuem alta taxa de mortalidade. Considerandose os retransplantes, o índice de letalidade sobe para $37,29 \%$. A maioria dos que foram a óbito eram do sexo masculino (72,92\%). O ano de 2009 foi responsável por $18,3 \%$ dos óbitos registrados, enquanto que o ano de 2014 apresentou $12,7 \%$ do total de óbitos, ou seja, houve uma redução de $30,61 \%$ na mortalidade ao longo de cinco anos.

Na Tabela 1, estão descritas as causas mais frequentes de óbito dos pacientes transplantados, que foram: causas gerais, choque séptico, causa técnica e não funcionamento primário do enxerto. As causas gerais, responsáveis por $34,93 \%$ dos óbitos, abrangeram: cardiovascular (91,25\%), cerebrovascular $(3,75 \%)$, insuficiência múltipla de órgãos $(3,75 \%)$ e renal $(1,25 \%)$. O choque séptico foi responsável por $16,59 \%$; causas técnicas, $4,8 \%$, e o não funcionamento primário do enxerto ficou com $3,93 \%$ dos óbitos. A taxa de letalidade do procedimento operatório estabeleceu-se em $35,93 \%$.

Tabela 1 -Distribuição dos pacientes transplantados segundo causa do óbito, considerando os pacientes retransplantados.

\begin{tabular}{lcc}
\hline \multicolumn{1}{c}{ Causa do Óbito } & N & IC (95\%) \\
\hline Causas gerais & $80(34,93 \%)$ & $(28,76-41,11)$ \\
Choque séptico & $38(16,59 \%)$ & $(11,78-21,41)$ \\
Causas técnicas & $11(4,8 \%)$ & $(2,03-7,57)$ \\
$\begin{array}{l}\text { Não funcionamento primário } \\
\text { do enxerto }\end{array}$ & $9(3,93 \%)$ & $(1,41-6,45)$ \\
Rejeição do enxerto & $8(3,49 \%)$ & $(1,12-5,87)$ \\
Não informado & $83(36,24 \%)$ & $(30,02-42,47)$ \\
\hline Total & $229(100 \%)$ & - \\
\hline
\end{tabular}

$\left(^{*}\right)$ Em "Causas gerais" ocorreram as seguintes causas de óbito: "Cardiovascular" com 73 casos $(31,9 \%)$, "Cerebrovascular" com três casos $(1,31 \%)$, "Renal" com um caso $(0,44 \%)$, "Insuficiência múltipla de órgãos" com três casos $(1,31 \%)$.

A trombose da artéria hepática foi a complicação pós-operatória responsável por cerca de 3,9\% dos transplantes no geral, sendo que essa condição apresentou associação com a alta mortalidade no procedimento.
Tabela 2 - Distribuição dos pacientes transplantados, segundo situação de óbito e diagnóstico, desconsiderando os retransplantes.

\begin{tabular}{lcccc}
\hline \multicolumn{1}{c}{ Diagnósticos } & \multicolumn{2}{c}{ Óbito } & Total & P \\
& $94(24,9 \%)$ & $57(26,9 \%)$ & $151(25,6 \%)$ & 0,0788 \\
\hline Hepatite C & $74(19,6 \%)$ & $36(17 \%)$ & $110(18,6 \%)$ & \\
Carcinoma hepatocelular & $74(19,6 \%)$ & $26(12,3 \%)$ & $100(16,9 \%)$ & \\
C irrose alcoólica & $33(8,7 \%)$ & $12(5,7 \%)$ & $45(7,6 \%)$ & \\
Cirrose criptogênica & $25(6,6 \%)$ & $16(7,5 \%)$ & $41(6,9 \%)$ & \\
Hepatite B & $16(4,2 \%)$ & $12(5,7 \%)$ & $28(4,7 \%)$ & \\
Hepatite autoimune & $10(2,6 \%)$ & $10(4,7 \%)$ & $20(3,4 \%)$ & \\
Câncer primário do fígado & $52(13,8 \%)$ & $43(20,3 \%)$ & $95(16,1 \%)$ & \\
Outros & 378 & 212 & 590 & \\
\hline \multirow{2}{*}{ Total } & $(100 \%)$ & $(100 \%)$ & $(100 \%)$ & \\
\hline
\end{tabular}

I - P: Valor P do Teste Qui quadrado de independência. Se $P<0,05$, então associação significativa

A partir do total de pacientes, $20,03 \%$ tiveram complicações pós-transplante. A sepse representou $23,57 \%$, trombose da artéria hepática 22,76\%, hemorragia 18,69\%, não funcionamento primário $8,94 \%$, desordens da coagulação $8,13 \%$ e trombose da veia hepática $2,43 \%$.

Os pacientes com 41 anos ou mais de idade foram mais frequentes no total de óbitos $(84,3 \%)$, sendo que nessa faixa etária o tempo de sobrevida foi de 2,24 anos e nas outras, esse valor não se diferenciou, de maneira evidente. Pacientes com até 20 anos apresentaram sobrevida de 2,49 anos e aqueles, no intervalo de 21 a 40 anos, foi de 2,13 anos.

No que se refere à causa de óbito e à sobrevida, as causas gerais apresentaram 0,99 anos de sobrevida, choque séptico, 0,69 anos; e rejeição do enxerto, 1,4 anos. O sexo masculino apresentou tempo de sobrevida, em anos, maior que o feminino $(2,28$ vs 2,12$)$. De modo geral, o tempo de sobrevida dos pacientes que não vieram a óbito teve média de 3,32 anos.

Figura 1 - Curvas de sobrevida dos pacientes segundo tipo de diagnóstico, desconsiderando os retransplantes.

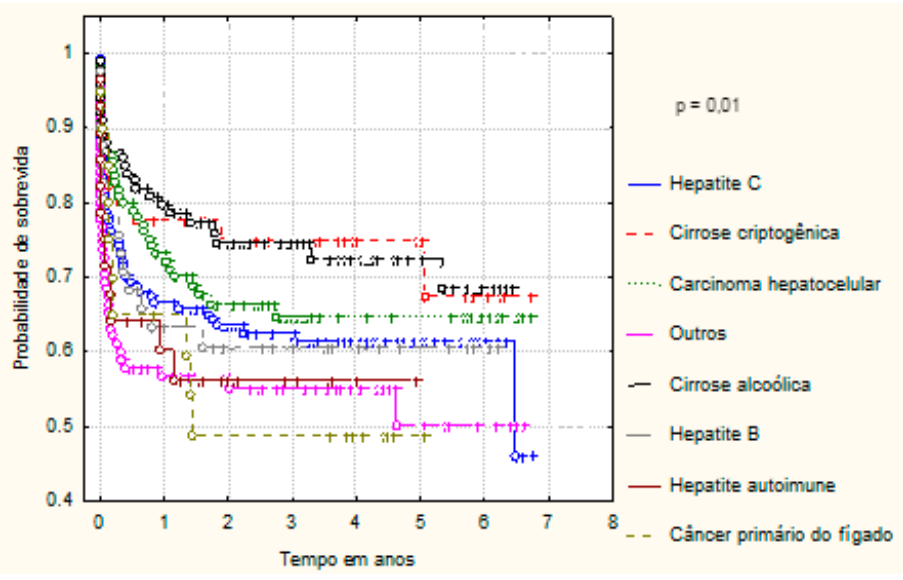


Conforme a Figura 1, houve diferenças significativas no tempo de sobrevida,comrelaçãoaotipodediagnósticodospacientes $(\mathrm{P}<0,05)$. A cirrose alcoólica e a cirrose criptogênica foram os diagnósticos que apresentaram tempo de sobrevida estatisticamente maior, ao passo que o câncer primário do fígado apresentou tempo menor. Pode-se ainda dizer que os outros diagnósticos apresentaram tempos de sobrevivência intermediários. A probabilidade de sobrevida geral, desconsiderando os pacientes retransplantados, em um ano foi de $67,94 \%$ e, em cinco anos, $60,70 \%$.

Tabela 3 - Tempo de sobrevida e probabilidades de sobrevida acumulada dos pacientes transplantados, desconsiderando os retransplantes.

\begin{tabular}{cc}
\hline $\begin{array}{c}\text { Tempo de sobrevida } \\
\text { (em anos) }\end{array}$ & $\begin{array}{c}\text { Probabilidade de sobrevida } \\
\text { acumulada }\end{array}$ \\
\hline 1 & $67,94 \%$ \\
2 & $64,82 \%$ \\
3 & $63,00 \%$ \\
4 & $61,70 \%$ \\
5 & $60,70 \%$ \\
\hline
\end{tabular}

\section{DISCUSSÃO}

Neste trabalho, foi possível observar maior prevalência de transplantes hepáticos no sexo masculino, representando $74,91 \%$ do total dos pacientes, apresentando consonância com outros estudos da literatura, que demonstraram $81 \%$ e $77 \%$ de transplantes em homens. ${ }^{9-11}$ Esse resultado deve-se provavelmente à maior taxa de etilismo dos homens em relação às mulheres, além da maior prevalência de usuários de drogas injetáveis e também por apresentarem atividade sexual mais promíscua, sofrendo, dessa forma, maior exposição a doenças virais e hepatopatia alcoólica. ${ }^{12}$

A média de idade obtida no estudo foi de 52,23 anos, tendo sido maior para os homens. Essa média vai de encontro aos achados de outros trabalhos, corroborando a evolução crônica e lenta das doenças hepáticas. ${ }^{13}$ Quanto à procedência dos pacientes, $77,5 \%$ tinham origem em Santa Catarina e $22,5 \%$ eram de outros estados do Brasil. Daqueles com origem em Santa Catarina, 14,7\% eram de Blumenau; $10,29 \%$, de Florianópolis e $8,4 \%$, de Criciúma. Comparando-se a estudo já realizado nesta instituição, foi possível observar aumento no número de pacientes procedentes de outros estados. ${ }^{9}$ Esse achado pode ser explicado pelos avanços nas técnicas cirúrgicas e na imunossupressão, que proporcionaram maior desenvolvimento do transplante ao longo dos anos, aumentando a abrangência de indivíduos submetidos ao procedimento. ${ }^{4}$
A indicação de transplante mais prevalente no trabalho foi para cirrose por Hepatite $\mathrm{C}$, conforme publicado por outros autores, ${ }^{10}$ com $25,6 \% \quad(n=151)$, seguido de carcinoma hepatocelular com 18,6\% ( $n=110)$ e cirrose alcoólica com 16,9\% $\quad(n=100)$, do total de pacientes. A prevalência de carcinoma hepatocelular foi equivalente à encontrada em um centro no Brasil. ${ }^{14}$ Porém, considerando-se os pacientes com diagnóstico de hepatocarcinoma infectados pelo vírus $C$ e os com infecção desse vírus de forma isolada, totalizam-se $34,85 \%$ do total de transplantes hepáticos. Esse dado foi estabelecido de forma similar ao estudo norteamericano, no qual foi encontrado o vírus $\mathrm{C}$ como responsável por $35-40 \%$ de todos os casos..$^{15}$

Atrombose deartériahepática, que estásignificativamente associada à mortalidade do transplante, apresentou prevalência aproximada à literatura em relação ao total de transplantes $(3,49 \%){ }^{16}$

Do tempo de permanência em UTI, 70,4\% dos pacientes permaneceram de zero a cinco dias e $18,2 \%$ permaneceram de seis a 10 dias. Além disso, o estudo revelou que, quanto maior o tempo de permanência em UTI, menor é a sobrevida dos pacientes. Esse resultado foi equivalente ao encontrado em um estudo semelhante realizado no Brasil. ${ }^{17}$

A partir do total de pacientes, 20,03\% tiveram complicações pós-transplante. Das complicações, houve discordância da literatura, que demonstrou prevalência de $4-12 \%$ de trombose de artéria hepática, $7-15 \%$ de hemorragia, 4-6\% de não funcionamento primário. ${ }^{18} \mathrm{~A}$ prevalência de trombose de veia hepática mostrou-se em equivalência com outro estudo, que encontrou essa complicação em 1-2\% dos transplantes hepáticos. ${ }^{19}$

Das causas de óbito, as mais frequentes foram: causas cardiovasculares $(31,87 \%$ do total de óbitos) e choque séptico, com $16,59 \%$ do total de pacientes que foram a óbito. Esses resultados foram divergentes de estudo semelhante, em que óbito por causas cardiovasculares representou $14,9 \%$ do total. ${ }^{20}$ Também, houve redução no número de casos por choque séptico em relação ao estudo anterior, ${ }^{9}$ que obteve essa causa em metade dos óbitos dos pacientes transplantados. Isso se deve provavelmente à melhoria dos cuidados perioperatórios, às novas técnicas cirúrgicas, à imunossupressão clínica e à melhor gestão de doadores. ${ }^{1}$

Gênero, idade, retransplante e etiologia da doença são variáveis importantes no impacto da sobrevida dos pacientes. Pacientes com menos de 30 anos resistiram mais, enquanto que os com mais de 55 anos demonstraram resultados piores. A média de idade da pesquisa em questão foi de aproximadamente 53 anos, o que pode ter corroborado para baixa taxa de sobrevida. ${ }^{20}$ 
$\mathrm{Na}$ classificação MELD, $70,28 \%$ dos pacientes obtiveram pontuação acima de 18 , o que também foi relatado em estudo semelhante. ${ }^{21}$ No presente estudo, quando comparado o tempo de sobrevida dos pacientes transplantados com o MELD, não foram encontradas diferenças significativas, com valor de $p>0,05$ no Teste $t$ de Student. Esse achado foi semelhante ao encontrado em outros estudos que compararam essa classificação com a sobrevida após o transplante hepático, e sugeriu que o escore MELD não foi um bom preditor de sobrevivência após o procedimento. ${ }^{11,22}$

A cirrose alcoólica foi o diagnóstico que apresentou maior probabilidade de sobrevida no primeiro ano, com valor aproximado de $80 \%$, assim como descrito na literatura, que constatou $86 \%$ de probabilidade de sobrevida. ${ }^{23,24}$ Devido à porcentagem majoritária de pacientes com realização de transplante por etiologia viral, a sobrevida dos mesmos é naturalmente menor que a dos estudos com etiologia alcoólica. Em seguida, o diagnóstico de cirrose criptogênica obteve probabilidade de sobrevida de $77 \%$, divergindo dos resultados encontrados em outras pesquisas. ${ }^{23,25}$

O tempo de sobrevida dos pacientes em um e cinco anos foi semelhante ao já publicado na literatura, os quais foram de, respectivamente, $69,4 \%$ e $62,6 \% .13$ Outro trabalho brasileiro relata o tempo de sobrevida em um e três anos, os quais foram de, respectivamente, $68,5 \%$ e $65,5 \%$, sendo que a média de tempo de sobrevida dos pacientes transplantados foi de 4,29 anos, corroborando com o estudo em questão. ${ }^{26}$

\section{CONCLUSÃO}

Os resultados encontrados neste estudo apresentaramse em sua maioria em consonância com outros demonstrados na literatura, sendo o perfil dos pacientes do sexo masculino, na sexta década de vida, oriundos principalmente do estado de Santa Catarina, porém foi observado aumento no número de pacientes com procedência de outros estados. A indicação de transplante mais prevalente foi cirrose por Hepatite $C$, e a principal causa de mortalidade foi por origem cardiovascular, divergindo do estudo anteriormente realizado nesta instituição. Também diferente da publicação anterior desse serviço, o presente estudo não evidenciou diferença significativa entre o escore MELD. A taxa de sobrevida geral, desconsiderando os pacientes retransplantados, em um ano foi de $67,94 \%$ e em cinco anos, 60,70\%.

\section{ABSTRACT}

Purpose: To assess patients submitted to liver transplantation in a unique transplantation center of the Hospital Santa Isabel in Blumenau/SC - Brazil, between January 2009 to May 2015. Methods: It is a study retrospective cohort type, in which the patient must be submitted to liver transplantation in the Santa Isabel Hospital of Blumenau/SC. The study was performed through data collection from the records of patients along their hospital stay for the transplant. It was assessed the epidemiological data (age, gender, city of origin, indication for the liver transplantation), MELD score, pos-transplant complications, causes of death and evolution of the transplant (occurrence of re-transplantation, survival and length of stay in Intensive Care Unit). Results: It was a total of 614 patients with average age of 52.23 years. Male sex was prevalent (74,91\%). As to the origin, $77.5 \%$ were from the State of Santa Catarina and $22.5 \%$ were from other states of the Federation. From the total patients, $55,86 \%$ had MELD higher or equal to 18 . The main indication for the transplant was cirrhosis due to hepatitis C (25.6\%) and hepatocellular carcinoma (18,6\%). Most patients $(70.4 \%)$ stayed between 0 and 5 days in ICU. The main causes of mortality was $35.93 \%$, without considering the retransplantation; and the main causes of death after the procedure along 6 years was general causes $(34.93 \%)$, having cardiovascular as prevalent cause $(91.25 \%)$, followed by insufficiency of multiple organs $(3.75 \%)$, cerebrovascular $(3.75 \%)$ and renal causes $(1.25 \%)$; septic shock (16.59\%), coagulation disorders $(5.24 \%)$, primary dysfunction (3.93\%), and causes not informed (35.8\%). The approximate survival rate in 1 and 5 years was respectively, $67.94 \%$ and $60.70 \%$. Conclusion: The study disclosed data similar to the literature, proving that this is an evolving service with many good results.

Keywords: Liver transplant, Survival Rate, Prognosis, Hepatic Failure, Liver Cirrhosis. 


\section{REFERÊNCIAS}

1. Åberg F, Gissler M, Karlsen TH, Ericzon BG, Foss A, Rasmussen A et al. Differences in Long-Term Survival Among Liver Transplant Recipients and the General Population: A Population-Based Nordic Study. Hepatology. 2015;61.

2. Dopazo C, Bilbao I, Castells LL, Sapisochin G, Moreiras C, Campos-Varela I et al. Analysis of adult 20-year survivors after liver transplantation. Hepatol Int. 2015 Jul;9(3):461-70.

3. Haque M, Scudamor $\mathrm{CH}$, Steinbrecher UP, Chung SW, Buczkowski AK, Siegfried ER. Liver transplantation: current status in British Columbia. Br Col Med. 2010;52:205-12.

4. Lodhi SA, Lamb KE, Meier-Kriesche HU. Solid Organ Allograft Survival Improvement in the United States: The Long-Term Does Not Mirror the Dramatic Short-Term Success. Am J Transplant. 2011 Jun;11(6):1226-35

5. Registro Brasileiro de Transplantes. Vínculo oficial da Associação Brasileira de Transplantes de Órgãos. 2007-2014 [citado em 2014]. Disponível em: http:// abto.org.br

6. Aberg F, Hockerstedt K, Roine RP, Sintonen H, Isoniemi $H$. Influence of liver disease etiology on long-term quality of life and employment after liver transplantation. Clinical Transplantation. 2012;26:729-35.

7. Gelson W, Hoare M, Dawwas MF, Vowler S, Gibbs P, Alexander $G$. The pattern of late mortality in liver transplant recipients in the United Kingdom. Transplantation. 2011;91:1240-44.

8. Merion RM. When is a patient too well and when is a patient too sick for a liver transplant? Liver Transpl. 2004;10:69-73.

9. Nogara MAS, Wiederkher J, Benghi RAC, Zalli M, Helena ETS. Avaliação dos transplantados hepáticos no estado de Santa Catarina no período de agosto de 2002 a janeiro de 2008. Jornal Brasileiro de Transplante. 2009;12:1142-46.

10.Russell RT, Feurer ID, Wisawatapnimit P, Lillie ES, Castaldo ET, Pinson CW. Profile of Health-Related Quality of Life Outcomes after Liver Transplantation: Univariate Effects and Multivariate Models. HPB : The Official Journal of the International Hepato Pancreato Biliary Association. 2016;10:30-7.

11. Silva OC, Nejo P, Cardoso N, Mente ED, Souza FF, Teixeira $A C$, et al. Prospective analysis of 44 consecutive liver transplants performed at a university hospital. Acta Cir. Bras. 2013;28:55-8.

12. Ferreira CT, Silveira TR. Hepatites virais: aspectos da epidemiologia e da prevenção. Rev. bras. Epidemiol. 2004;7:473-87.

13. Meirelles RFJ, Salvalaggio P, Rezende MB, Evangelista AS, Guardia BD, Matielo CEL, et al. Transplante de fígado: história, resultados e perspectivas. Einstein (São Paulo). 2015;13:149-52.

14. Freitas ACT, Parolin MB, Stadnik L, Coelho JCU. Carcinoma hepatocelular: impacto do tempo em lista e das formas de tratamento pré-operatório na sobrevida do transplante de fígado cadavérico na era pré-MELD em um centro no Brasil. Arq. Gastroenterologia [série online]. 2007 [citado em 29 fev de 2006]; 44(3):189-94.
15.Tsoulfas G, Goulis I, Giakoustidis D, Akriviadis E, Agorastou P, Imvrios G, et al. Hepatitis C and liver transplantation. Hippokratia. 2009;13:21115.

16.Akun E, Yaprak O, Killi R, Balci NC, Tokat Y, Yuser $\mathrm{Y}$. Vascular complications in hepatic transplantation: single-center experience in 14 years. Transplantation Proceedings. 2012;44:1368-72.

17. Lacerda CM, Melo PSV, Lucena O, Amorim A, Almeida HM, Pereira LB. Transplante de fígado em hospital universitário do Recife: resultados iniciais. Rev. Col. Bras. Cir. 2003;30:29-35.

18. Quiroga S, Sebastià MC, Margarit C, Castells L, Boyé $\mathrm{R}$, Alvarez-Castells A. Complications of Orthotopic Liver Transplantation: Spectrum of Findings with Helical CT 1. Radiographics. 2001;21(5):1085-102.

19. Itri JN, Matthew TH, Mitchell ET. Hepatic transplantation: postoperative complications. Abdom. Imaging. 2013;38:1300-33.

20.Schoening W, Buescher N, Rademacher S, Andreou A, Kuehn S, Neuhaus R, et al. TwentyYear Longitudinal Follow-Up After Orthotopic Liver Transplantation: A Single-Center Experience of 313 Consecutive Cases. American Journal of Transplantation. 2013;9:2384-94.

21. Lombardi AC, Mente ED, Sankarankutty AK, Souza FF, Teixeira AC, Cagnolati D, et al. Analysis of 83 consecutive liver transplants performed at a tertiary care reference hospital in the interior of the state of Sao Paulo. Acta Cir. Bras. 2011;26:530-34.

22. Batista TP, Sabat BD, Melo PSV, Miranda LEC, Fonseca OCL, Amorim AG, et al. Impact of MELD allocation policy on survival outcomes after liver transplantation: a single-center study in northeast Brazil. Clinics. 2011;66:57-64.

23.Iruzubieta P, Crespo J, Fábrega E. Long-term survival after liver transplantation for alcoholic liver disease. World journal of gastroenterology. 2013;48:9198-208.

24.Burra P, Senzolo M, Adam R, Delvart V, Karam V, Germani G, et al. Liver transplantation for alcoholic liver disease in Europa: a study from the ELTR (European Liver Transplant Registry). American Journal of Transplantation. 2010;10:138-48.

25.Adam R, Karam V, Delvart V, O’Grady J, Mirza D, Klempnauer J, et al. Evolution of indications and results of liver transplantation in Europe. A report from the European Liver Transplant Registry. Journal of Hepatology. 2012;57:675-88.

26. Haddad L, Cassenote AJF, Andraus W, de Martino $\mathrm{RB}$, Ortega NR, Abe JM, et al. Factors Associated with Mortality and Graft Failure in Liver Transplants: A Hierarchical Approach. Plos One. 2015;10. 